\title{
Infrared landmine detection and thermal model analysis
}

\author{
Piet B.W. Schwering ${ }^{a}$, Athina Kokonozi ${ }^{\mathrm{b}}$, Lawrence J. Carter ${ }^{\mathrm{c}, \mathrm{b}}$, \\ Henk A. Lensen ${ }^{a}$, Eric M. Franken ${ }^{a}$
}

${ }^{4} T N O$ Physics and Electronics Laboratory, P.O. Box 96864, NL-2509 JG The Hague, Netherlands, Schwering @ fel.tno.nl

${ }^{b} J R C-I S I S$, TDP unit, TP:272, Via E. Fermi 1, I-21020 Ispra (VA), Italy, athina.kokonozi(a'jrc.it

'University of Auckland, Department of Electrical and Electronic Engineering, 20 Symonds Street, Auckland, New Zealand,

li.carter@auckland.ac.nz.

\begin{abstract}
Infrared imagers are capable of the detection of surface laid mines. Several sensor fused land mine detection systems make use of metal detectors, ground penetrating radar and infrared imagers. Infrared detection systems are sensitive to apparent temperature contrasts and their detection capabilities are inversely proportional to the amount of background clutter generated by local surface non-uniformities. This may result in spurious detections, or even cancellation of true detections in a post classification process. Sub-surface mines can be detected when buried not too deeply. Furthermore, soil type and soil water content will influence the detection result. For this reason experiments in various soil types, including vegetation, and soil circumstances are essential for understanding and improving the infrared detection capabilities. We have performed outdoor experiments with different types of soil and weather conditions. Several examples are described and analyzed. Data analysis shows the strong correlation of apparent temperature with thermocouple gradients and solar energy, as well as a correlation of local standard deviation with these parameters. Model based temperature contrasts are predicted for several mines in sandy soils, and these are compared with infrared imaging apparent temperature measurements and thermocouple data. The comparison results are quite good but also show the complexity of the thermal infrared data, in particular due to infrared clutter, diurnal variations, and sky reflectance contritutions. Model predictions are made for the application of active heating methods also. Limitations of the model and potential future expansions based on evaluation of experiments are discussed. We discuss the potential use of modeling for therrnal infrared detection and sensor fusion applications.
\end{abstract}

Keywords: Mine detection, passive infrared, experimental data, thermal model comparison.

\section{INTRODUCTION}

One of the detection techniques for de-mining is based on thermal imagery. Different studies have been performed into the field of thermal infrared (TIR), both for measurements and model simulations (Uppsäl et al.', Şendur and Baertlein ${ }^{2}$ ) as well as for statistically optimizing infrared detection (Simard ${ }^{3}$ ). The TIR sensor will however in most cases be part of a sensor suite, in combination with a metal detector and a ground penetrating radar system. This requires the use of sensor fusion techniques to combine the individual sensor output streams (Schwering ${ }^{4}$ ). The sensor fusion process can be optimised by the employment of sensor models. The use of TIR sensor data has been shown at various success rates in different scenarios. In particular for surface objects TIR plays a major role. For sub-surface objects the use of other information is important. This could mean the use of other sensors in a sensor fusion process, as well as the use of predicted TIR features by modeling.

The present work describes the development and evaluation of a TIR model for the simulation of the thermal behaviour of mine-containing soil and the corresponding thermal IR imagery of the soil surface for de-mining purposes. This modeling is
done for several reasons. First, it done for several reasons. First, it provides a way to gain insight in the physical properties and mechanisms involved, without the time and budget consuming experimental sessions. The long-term goal however is to assist the fusion process of the TIR sensor with the other sensors. In this respect, one could think of the prediction of the plausibility of a specific detection demining a model-based control of the sensor or fusion parameters or an advice for a time-of-day selection for the optimum demining detection measurement. The aim is to have a model that predicts the use of the TIR sensor with good accuracy. We start with a simple model that can be compared to accessible TIR and quantitative meteorological data, and improve the cssential in on extensive testing and evaluation phases in an iterative process. Hence the use of experimental data is issential in this approach, as the features present in the data will influence the way ahead in the modeling. 



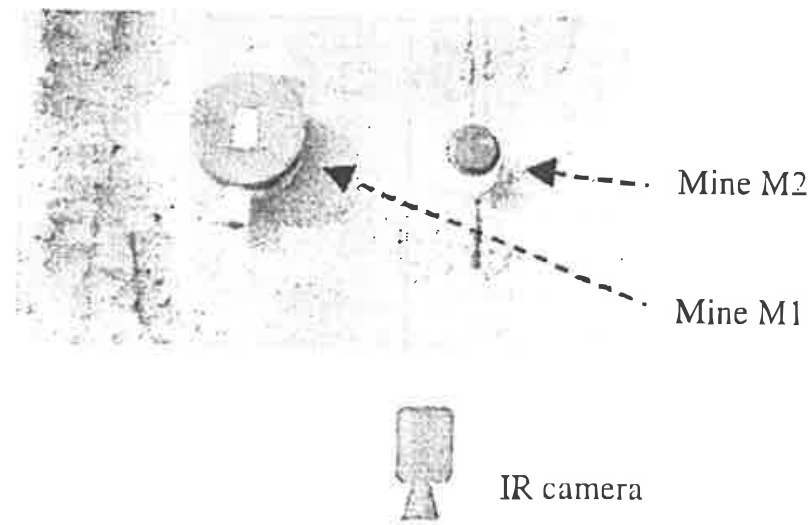

$1.5 \mathrm{~m}$

Above

MI

Below

M1

$\begin{array}{llr} & \text { Above } & 1.5 \mathrm{~m} \\ \text { M1 } & \\ \text { Below } & & \text { Above M2 } \\ \text { M1 } & \text { Below M2 }\end{array}$

Figure 2-A: Surrogate mines that were placed in JRC's test lane; positions before covering them with soil.

Figure 2-B: Configuration of the measurement set-up and the measurement area.

$2 \mathrm{~cm}$ Free Area

$\sim \mathrm{cm}$ Free Area

The soil type was sandy and the moisture level at $12 \%$ vol. A weather station was located at about $1.5 \mathrm{~m}$ from the test area that measured solar energy, air temperature, and wind speed. All the data (thermocouple readings, meteo data, TIR images), were collected simultaneously every 15 minutes. The TIR-images were recorded in two periods during 48 hours. The first lest period was on 27 November 2000 from 11:15 am until 16:15 pm, and the second day (28 $\left.8^{\text {th }}\right)$, from 11:00 am until 20:00 pm. The TIR-images were captured with an FLIR-Agema 570 camera with spectral range: $7.5-13 \mu \mathrm{m}$ in a vertical, down looking direction. At 14:00 local time a local shadow was introduced over the test field by the meteo station.

\section{THERMAL INFRARED MODEL DESCRIPTION}

Infrared radiation of objects is caused by their thermal behavior and their reflective characteristics. A good description of the thermal process is given in Jacobs. We have based our thermal infrared model on the description given by Jacobs in this reference. The aim of the TIR model is to predict surface temperatures for the actual meteorological condition. The input parameters for the model are the material of the soil surface layer and of the mine, the meteorological conditions and the initial temperature profile of the surface layer. The model should be able to predict both 'natural' temperature effects, but must also be capable of calculating the effect of actively induced heating, by means of optical illumination, micro-waves or blown hot air. In this way we are able to predict the performance of thermal infrared imagery when used in combination with an active heating source. In literature, several models are reported predicting the temperature behaviour of the ground. Most models solve the heat equation

$$
\frac{\partial T}{\partial t}=\kappa \frac{\partial^{2} T}{\partial z^{2}}+\vartheta_{\text {source }}
$$

In one dimension, with $z$ the depth beneath the surface, $\kappa$ the thermal diffusivity (in $\mathrm{m}^{2} \mathrm{~s}^{-1}$ ) and $\vartheta_{\text {source }}$ the internal heat ') urces (in $\mathrm{K} \mathrm{s}^{-1}$ ), if present. $T$ is the temperature in Kelvin and $t$ the time in seconds. In this work a one-dimensional model
is used. This approach enables a fast evaluation of the effect of model parameters. Furthermore, this enables a comparison 
of the modeling results with experimental data, leading to model validation and a better understanding of the experimental results. The final objective is to incorporate the validated model in feature fusion algorithms (Schwering ${ }^{4}$ ). In order to do so, a continuing step would be to predict infrared imagery from the mine and surface clutter.

In the model, two temperature profiles are calculated, for a surface layer composition with and without a buried mine present. The one-dimensional treatment implies that only vertical energy transport occurs. In other words, the surface is thought to be homogeneous. This assumption in the model, for the case with a mine, is only valid for (the centre of) large mines. When needed, a three-dimensional calculation can be inserted at a later stage to investigate the effects on the model performance. At present we emphasise investigating the major areas for model upgrade. These are described in the discussions in section 5 .

The following meteorological effects are implemented in the model: short-wave irradiance absorption $Q_{\text {shornvave, }}$ long-wave irradiance absorption $Q_{\text {longwive }}$, heat exchange by convection $Q_{\text {convection, }}$ and surface emittance $Q_{\text {emiltance. }}$ The model implementation is described in detail in Jacobs ${ }^{7}$. The combined effect of the meteorological conditions is therefore given by

$$
Q_{\text {nett }}=Q_{\text {shornvave }}+Q_{\text {longwave }}+Q_{\text {convection }}-Q_{\text {emittance }}
$$

Note that latent heat exchange is not implemented. This is a model limitation, especially for active heating simulations. To study active heating in the future, the model is expanded with a thermal heating source, such as optical illumination and air heating of the surface, as well as micro-wave heating of the soil. When heating by means of hot air is applied in the model, during the heating phase the meteorological data is replaced by artificial meteorological data describing the hot air heating. For each object and soil material the following physical coefficients must be known:
- density
- specific heat
- thermal conductivity
- optical reflectivity

$$
\begin{aligned}
& \text { in } \mathrm{kg} \mathrm{m}^{-3} \\
& \text { in } \mathrm{J} \mathrm{kg}^{-1} \mathrm{~K}^{-1} \\
& \text { in } \mathrm{J} \mathrm{m}^{-1} \mathrm{~K}^{-1} \mathrm{~s}^{-1} \\
& \text { in fraction } 0-1 \text { (only for surface material) }
\end{aligned}
$$

Simulations based on active heating are given in the discussion section. Note that these active heating approaches also require additional material characteristics on reflectivity and absorption.

\section{INFRARED DATA ANALYSIS}

In JRC's TIR data, the apparent temperatures for the mines have been measured from the images by calculating the mean values of the temperature for areas with diameter 14 pixel and 7 pixel at the location of the big and the small mine respectively. The mean value for the object free area was calculated in a rectangular area between the mines, and is larger than the mine area (figure 3 ). The contrast was calculated as the difference between the apparent temperature for the mine area and the one for the free area (FA).


Figure 3: TIR image from the $27^{\text {th }}$ of November 2000 at 13:00, with the positions of the mines and the mine free area indicated (right). The indicated temperatures are the mean apparent temperatures of the respective areas. 
Table 1 gives correlation coefficients of the two mine areas for the standard deviation of the apparent temperatures. From this table we notice a dependence of this standard deviation on the solar energy, on the temperature difference between the area above the mines and the one below them, and on the apparent temperature of the corresponding areas.

\begin{tabular}{|l|c|c|}
\hline 28 Nov. 2000 & STD for M1 area & STD for M2 area \\
\hline Solar Energy & 0.694 & 0.835 \\
\hline Apparent temperature & 0.678 & 0.896 \\
\hline$\left(T_{\text {above }}-T_{\text {below }}\right)$ the mine & 0.662 & 0.913 \\
\hline
\end{tabular}

Table 1: Correlation coefficients between the solar energy, the apparent temperature, and the temperature difference above and below the mines with the standard deviation STD for the areas of interest above the mines.

The change of the standard deviation STD over the areas of interest could affect the detection results. We noticed that there were cases where the suspect area was indicated by a larger STD than its surroundings, rather than by the apparent local contrast. Looking at the resulting thermal images we conclude that the observed thermal contrast for the areas above the mines is not the same for the entire observation time. It can be positive, negative or zero, depending on the time of day. The process is affected by the way that the soil exchanges energy with the surroundings. At 13:45 hours of the $28^{\text {th }}$, there is a shadow of an object interfering with the measurements. For the same day, after 14:00 hours in the afternoon, the test area was covered by the shadow of the meteo station which was very close $(\sim 1.5 \mathrm{~m})$ to the test lane. This has produced a large decrease for the apparent temperature for the corresponding testing period.

For the larger mine M1 a clear positive contrast is observed in figure 3. No clear image of the small mine M2 is obtained. The small mine seems to cause the same disturbance to the heat flow, but it can not produce an observed thermal contrast on the surface. That leads us to the assumption that the geometry and the thermal properties (heat conduction and specific heat) of the surrogate mine also affect the results. The presence of the buried surrogates influences the resulted thermal pattern.

\begin{tabular}{|c|c|c|}
\hline 28 Nov 2000 & Apparent temperature for $M I$ & Apparent temperature for $M 2$ \\
\hline Solar energy' & 0.9493 & 0.9457 \\
\hline Air temperature & 0.5179 & 0.4864 \\
\hline Wind speed & 0.1397 & 0.1040 \\
\hline$\left(T_{\text {uldriet }}-T_{\text {belour }}\right)$ in the ground & 0.9745 & 0.9816 \\
\hline
\end{tabular}

Table 2: Correlation coefficients berween the solar energy, the air temperature, the wind speed and the temperature difference above and below the mines with the apparent temperatures for mine MI and mine M2.

In table 2 we present the correlation between meteorological parameters and apparent mine temperatures. We note a strong dependence of the apparent temperatures on solar energy and with the thermocouple gradient measurement in the ground.

During the solar heating phase (solar energy $>150 \mathrm{Wat} / \mathrm{m}^{2}$ ) the area above the mines appears warmer than the object free area (see figure 3). The temperature there is also higher than the temperature below the mines. During the afternoon cooling phase (solar energy $<150 \mathrm{Wat} / \mathrm{m}^{2}$ ) the opposite is the case. The temperature below the mines is higher than the one above them. This temperature difference (recorded by the thermocouples) between the locations above and below the surrogate These experimental entire test period seems to be higher than the one between the $2 \mathrm{~cm}$ and $6 \mathrm{~cm}$ for the object free area (FA). heat flow. During the heats support the interpretation that the presence of the objects in the ground causes impedance to the top layer of the mine heating phase this produces a hot spot on the surface over the mine area, since the area between the the heat flows from the ground to he the warmer than the corresponding one at the object free area. In the cooling phase between the mine and the surface and surface and it is blocked from the bottom layer of the mines. This cools the area flow is depending on the georface and produces a cool spot on the surface over the mine area. The impedance of the heat is depending on the geometry of the buried object and its thermal properties (heat conduction and specific heat).

\section{DISCUSSION}

In this section we discuss the comparison between the thermal infrared model and the various experiments. As we know that a [ull validation of the model will take a large effort, and we already know that the present version of the model has a number of limitations, the present evaluation is aimed to show the model validity domain and further model limitations. 
Hence the model improvement will strongly depend on the evaluation on the test results. In particular for the infrared imagery, model development should go hand in hand with experimental verification of the results. We also aim to show where infrared model knowledge is capable of assisting the detection process with simulated predictions. The discussion is based on model and experimental comparison in the case of passive infrared sensing. At the last part of the present section model predictions are performed for infrared detection for active illumination of the soil. The data described in section 2 is used to investigate the model performance for predicting the soil temperature at different depths and at the surface for soils with and without surrogate mines.

\subsection{Temperature modeling of the diurnal cycle of 3 August 1998}

The TIR modeling of the data on 3 August 1998 is given in figure 4. This date was a nicely warm summer day, rith abundant solar irradiation (see also figure 1). The graphs in the figure show the surface temperatures of, and contrasts between, a mine filled with TNT substitute material $(5 \mathrm{~cm}$ thick) and the surrounding soil, based on modeled and experimental data. The mines are buried at depths from $-5 \mathrm{~mm}$ to $5 \mathrm{~cm}$. The experimental data are thermocouple data recorded at different depths in the soils and on the buried mines. Note that the initial temperature difference for the model situation is zero by definition, since the initial temperature profile of the soil with and without mine is assumed to be similar. At least part of the differences from 00:00 - 10:00 hour is due to this incorrect temperature initialization. The temperatures and contrasts for the outdoor sandy soil and for the mines are calculated for a 24-hour period, starting at midnight, using the meteorological conditions of 3 August 1998.

We first analyze the empty sandy outdoor soil. Figure 4-A, B and C contain the modeled and recorded thermocouple soil temperatures for 0,5 , and $10 \mathrm{~cm}$ depths, respectively. Note the good correlation between model and experiment, except at the measured peak. Deviations of the model from experimental data of up to +2 degrees occur only at the peak of the curve in the afternoon. The morning temperature dip before sunrise is visible in the model with a delay of about one hour with respect to the experimental data. Note the reduced swing and delayed effects for deeper thermocouple positions.

Beside empty soil we also analyzed mine temperatures and mine contrasts for different depths. Figure 4-D presents model and experimental surface temperature contrast data for a very shallow mine (with just a minute sand layer over it, modeled as $5 \mathrm{~mm}$ ). Note that contrast difference deviations are at maximum of the order of 4 degrees before sunrise and approximately +4 degrees at the temperature peak. Figure 4-E shows the modeled surface temperatures for different mine depths and for the empty soil. The same mine is modeled and temperature variations occur of the order of up to -5 degrees before sunrise, up to +5 degrees during daytime, compared to the case of empty soil. As expected, the temperature increase is stronger for shallower mines, while deeper buried mines show a stronger attenuation effect. Note the potentially strong influence of the mine depth on the temperature contrast. Figure 4-F shows the simulated temperature contrast for two mine depths of 0.5 and $5 \mathrm{~cm}$. We again observe the stronger influence of the shallower mine to the temperature and the stronger attenuation of the deeper mine. This difference is of the order of -2 degrees before sunrise, up to +4 degrees during the peak of the global irradiance. The shallow mine interacts more strongly with the global irradiance. We also observe the time delay in heating and cooling of the deeper mine compared to the shallower one. For the morning dip the delay is about 1 hour, while the delay is approximately 2 hours for the temperature decrease in the afternoon, although the latter delay is less clear. Obviously the gradients differ for the two mines at different depths. Furthermore, the temperature contrast zerocrossing moment at the surface (indicated in figure 4-F with vertical dashed lines) changes with the depth of the mines ( 1.4 hour delay for the $4.5 \mathrm{~cm}$ deeper mine). This indicates that at different times (sometimes hours after sunrise or sunsel). mines can still be invisible due to the poor contrast. The diurnal temperature curve at a specific depth below a mine is independent of the actual mine depth (data not shown).

General conclusions from the graphs in figure 4 are that the infrared model predicts the surface and depth temperatures (down to $10 \mathrm{~cm}$ ) quite well, with a maximum error of about 2 degrees at the maximum of the temperature curve. The data of figure 4-F shows nicely the problem with using TIR images for mine detection without additional heating: the moments at which mines buried at different depths show a thermal contrast relative to the background differ considerably. The observed delays between the zero crossings (at which times the mines cannot be discerned using TIR imaging) most likely vary with meteorological conditions (solar illumination) and soil type (thermal conductivity). When passive TIR imaging is used for mine detection, sufficiently accurate modeling will have to be: applied to rule out the danger of missing mines due to meteo induced low contrast and differences in contrast, and clutter. Artificially simulated TIR imaging, using for example microwave heating, may be a way of avoiding this problem. 



Figure 4: A-C: Modeled and observed soil temperature at depth 0 (surface), 5 and $10 \mathrm{~cm}$, for outdoor sandy soil at 3 August 1998, respectively (thick line is experimental thermocouple data, thin line is model data). D: Surface temperature contrast tor a mine, buried in outdoor sand just beneath the surface, as obtained from model (thick solid line) and experimental (thin (ntted line) data for 3 August 1998. E: Modeled surface temperatures at 3 August 1998 above mines buried in sand at arious depths from 0.5 to $5 \mathrm{~cm}$ (thin lines) and for mine free surface (thick line). F: Modeled surface tenperacure contrast "with minus without a mine present") for mines at depths of 0.5 and $5 \mathrm{~cm}$. In all graphs, the time scale starts at midnight. 


\subsection{Temperature modeling of the diurnal cycle of 23 October 1998}

This day is a grey, yet dry, autumn day, without much sunshine. The meteorological conditions are presented in figure $I$ in section 2. Figure 5 shows the surface temperature difference between a mine filled with TNT substitute material $(5 \mathrm{~cm}$ thick) and the surrounding sandy soil, based on modeled and experimental data. In both cases, the mine is just covered with soil (for the model the minute experimental depth is assumed to be $5 \mathrm{~mm}$ ). Again, the initial temperature difference for the model situation is zero by definition, since the temperature profile of the soil with and without mine is taken to be similar at $t=0$. From the contrast figure below we conclude that the temperature is modeled quite well. Note that the contrast values are much smaller than in the sunny summer case. The maximum difference between model data and experimental data of 0.5 degrees occurs in the afternoon. Note that also these deviations between model and experiment are much smaller than in the summer case.

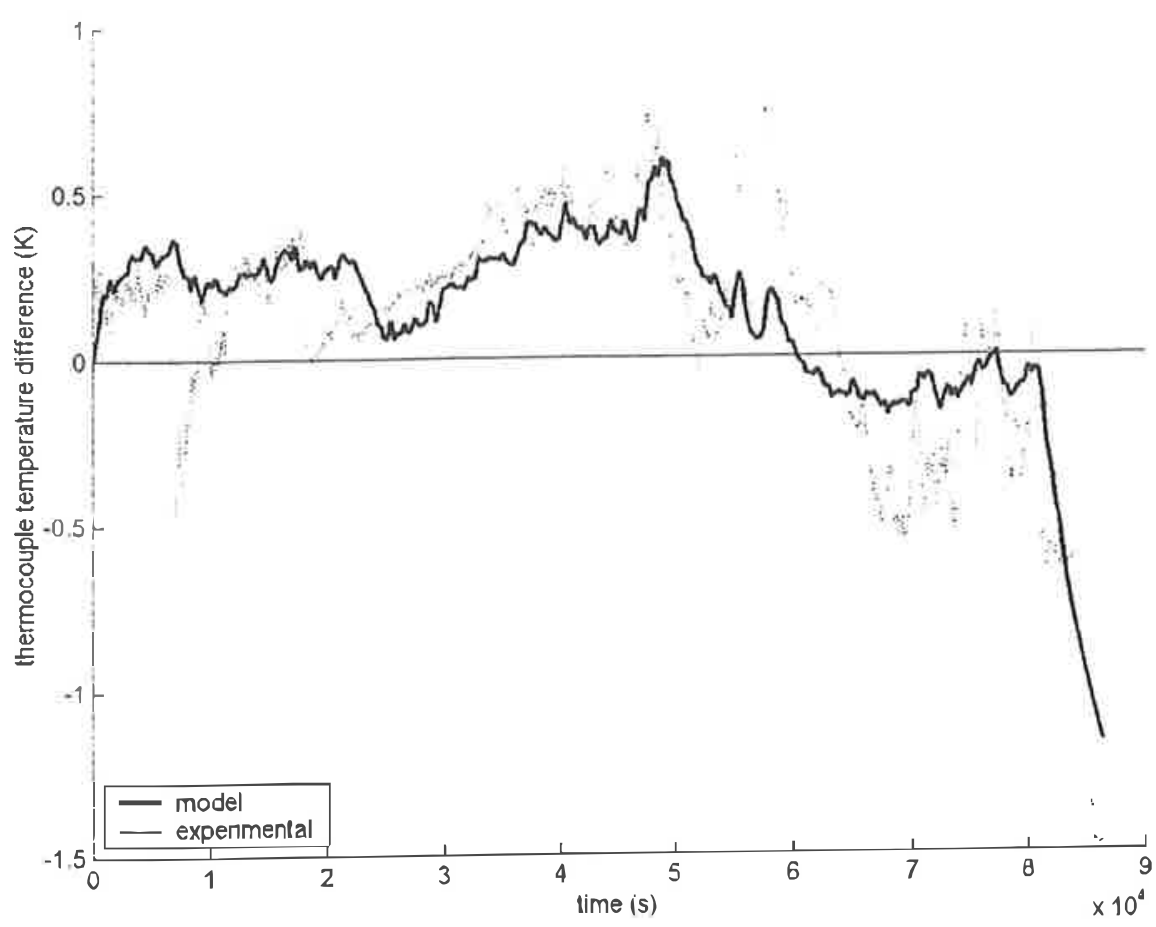

Figure 5: Surface tenperature contrast for a mine, buried in outdoor sand, just beneath the surface, as obtained from experimental (thin line) and model data (thick line) for 23 October 1998.

\subsection{Thermal infrared imagery recordings}

We have also compared the infrared model with the passive imagery recordings described in section 2 . The image analysis is described in section 4 and model comparisons were performed in order to understand the major complexities during the measurements. In figure 6 below we present a comparison between the modeled infrared and apparent surface temperature data at the free area (defined in section 2.2) generated from the thermal infrared imagery. The surface temperature data derived from the imagery shows a fast decay at 14:00 hours because of the shadow of the meteo station over the test area. This is modeled by setting the solar global irradiance to zero. This could be considered as a lower limit as unrelated global irradiance measurements at TNO (figure 1) show values of the order of $10-40 \mathrm{Watt} / \mathrm{m}^{2}$ in cases of overcast during the fall. The true remaining global irradiance values would depend on the actual ratio between direct and diffuse radiation. Recorded global irradiance and air temperature are also given in the figure, as well as apparent mine temperatures. The model redictions follow quite well the experimental output for the apparent temperature variation (extreme errors could be -2 and +4 degrees respectively for those days). Note that the apparent temperature decay caused by the shadow is stronger on the warmer day, and that this decay is too steep for the model to follow. From figure 6 we observe that the time scale of 15 minutes is too large for the changes that occur in the meteo parameters. The effect of this undersampling is visible in the decay structure after each point in the modeled graphs. Furthermore actual measured temperature fluctuations are stronger than model predictions. In the late afternoon the low apparent surface temperatures are respectively 3 and 5 degrees below 
the $2 \mathrm{~cm}$ deep thermocouple temperature (not given in the graphs). These numbers are comparable to the maximum deviations in the model values. This could be consistent with the presence of more cold sky reflection during the measurements than predicted in the models (see Jacobs ${ }^{7}$ ). Note that the 28 November data were recorded with clear sky conditions, while on 27 November there were clouds present. Hence the cold sky contribution to the apparent temperature would be less that first day. The model does not take these differences due to sky reflection into account in the apparent iemperature calculation. We conclude that the model performs worse for the thermal imaging data than for the thermocouple data. Further studies into the comparison between model output and apparent temperature mine contrast from thermal imaging would benefit from incorporation of more accurate cold sky models. These results show that surface modeling is more complicated than sub-surface modeling.




D APPARENT TEMPERATURE VARLATTON \& METEO DATA

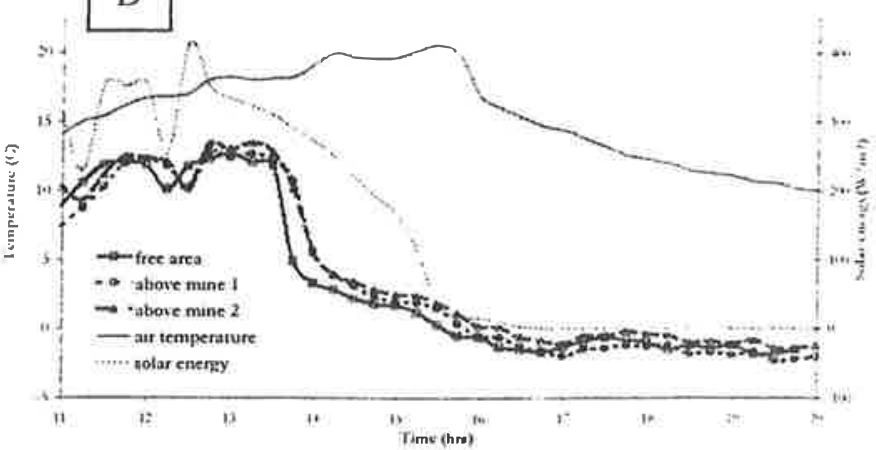

Figure 6: Comparison between modeled and experimental (based on IR imagen') surface apparent temperatures on 27 (A) and 28 November 2000 (C) for the mine free area. Panels B and D indicate the experinentally obtained apparent temperatures (based on IR imagen') of the surface above the mines and the mine free area as well as the air temperature und solar energy (right axis) for $27^{\text {th }}$ and $28^{\prime \prime \prime}$ Nov. 2000, respectively.

\subsection{Modeling active heating methods.}

The basic active heating methods compared in this section are heating by optical illumination and heating by micro-wave. Jptical illumination experiments, such as heating with lamps, have been executed at the TNO test lane and first results are lescribed in de Jong and de Jong ${ }^{8}$. With this method only the surface is heated by a total energy of about $1400 \mathrm{Watt} / \mathrm{m}^{2}$ for ome 10 minutes while penetration of the soil is modeled as described in section 3 . In the case of micro-wave heating deeper ayers are also heated (see e.g. Carter et al. ${ }^{9}$ ). The absorbed power is assumed to decrease exponentially with depth and is i.s. Vote that for micro-waves much higher power densities are reported (Khanna et al. ${ }^{10}$ ).

The approach is to model the temperatures in order to find differences in apparent temperatures in different situations. These ifferences, when available, could then be utilized in the detection stages in the processing. Figure 7 shows the simulated urface temperature for optical illumination and microwave illumination during the heating and the relaxation phase. 

Calculations for heating by optical illumination with mines at different depths show a steep decreasc in maximum achievable contrast with depth. For shallow mines maximum temperature contrasts of $8.3,4.5,2.5$ and 1.0 degree are reached after about $600,650,850$ and $3000 \mathrm{~s}$ for mines buried at $0.1,0.5,1.0$ and $2.5 \mathrm{~cm}$, respectively. For deeper mines the maximum temperature contrasts are much lower and are not reached within the calculated time-frame. This steep decrease with mine depth shows the complexity of using passive infrared detection techniques for the detection of buried objects, as well as the importance of this technique for the detection of shallowly buried mines.

For optical heating the temperature contrast (mine versus without mine) as a function of time is indicated in ligure 8 . This ligure is explained qualitatively as follows. The thermal conductivity of TNT substitute material is much lower than for the surrounding soil. Thus, when the energy is transferred towards lower depths during the heating stage, the energy 'gets stuck' when it reaches the mine top surface. Until the energy reaches the mine, there is no temperature difference between the situation with and without mine (from the figure it can be observed that it takes over $500 \mathrm{~s}$ for the energy 10 (ravel $10 \mathrm{a} 5 \mathrm{~cm}$ Jepth). The mine upper surface acts as an energy trap, and the soil above it will consequently attain a higher temperature ihan the surrounding soil. Consequently, the back-transfer of energy towards the surface will be enhanced when the mine is present. Thus, in the presence of a mine, the surface temperature will be higher than without a mine present. The mine induces a positive contrast in the TIR image.


Figure 8: $2 D$ representation of the temperature difference (intensity indication in $K$ ) between the situation with and without a mine as a function of time (mine depth $5 \mathrm{~cm}$, mine thickness $12 \mathrm{~cm}$, optical heating duration $600 \mathrm{~s}$, mine material TNT, roil material sand $17 \%$ moisture (Carter et al. $\left.{ }^{9}\right)$ ). The right panel shows a schematic outline of soil composition.

\section{CONCLUSIONS}

The relatively simple model defined in this paper simulates thermocouple recordings well in sunny and gray solar llumination conditions, at the surface as well as at depths up to $10 \mathrm{~cm}$. In particular in well-defined meteorological conditions the model predictions are quite good. A comparison with surface derived thermal imagery still shows a ubstantial difference in apparent temperatures of 2-4 degrees for a mine free area. The surface modeling results in larger nodel errors than sub-surface modeling. Part of this could be due to the effects of infrared surface clutter, and stronger neteorological variations. The strong effect of surface texture on the data analysis and on TIR processing is described, as ell as the correlations between apparent temperature and standard deviations with meteorological parameters.

urther modeling and data analysis is needed to fully understand these TIR data. Future model improvements are expected the field of soil moisture content, optical reflectivity, and evaporation and transpiration (see Lensen et al. ${ }^{11}$ ). Other model inprovements will be based on the evaluation of the different test results, which could comprise expansion to multiimensional, modeling of sky reflection, and active heating and shadowing effects. Furthermore, the use of local neteorological data, close to the test lane, might be preferable over standard meteorological data. 
The main use of the TIR models is to predict defection performance. In order to optimize the detection strategy, the model can be used to predict TIR performance, also when applying active illumination such as micro-waves, and to support sensor fusion processing by adapting TIR weights in the fusion process, based on the model outputs. The strong dependence and variability of passive TIR and temperature data as presented in this paper suggest benefits from modeling for predicting ideal moments for detection measurement. Furthermore the use of active heating and active cooling, such as micro-wave heating and shadowing, should be considered as both the heating and cooling phases show the strongest temporal thermal gradients.

\section{ACKNOWLEDGEMENTS}

We kindly acknowledge useful inputs from and discussions with Brian Hosgood and Wim de Jong.

\section{REFERENCES}

1. M. Uppsäl, LM. Petterson, M. Georgson, S. Sjökvist, Temporal IR contrast variation of buried land mines, in 'Detection and Remediation Technologies for Mines and Minelike Targets V', Proceedings SPIE-Orlando Vol. 4038 pp 146-155, April 2000

2. I.K. Sendur, B.A. Baertlein, Numerical Simulation of Thermal Signatures of Buried Mines over a Diurnal Cy'cle, in 'Detection and Remediation Technologies for Mines and Minelike Targets V', Proceedings SPIE-Orlando Vol. 4038 pp 156-167, April 2000

3. J.-R. Simard, Improved Landmine Detection Capability (ILDC): Systematic approach to the detection of buried mines using passive IR imaging, in 'Detection and Remediation Technologies for Mines and Minelike Targets'. Proceedings SPIE-Orlando Vol. 2765 pp489-500, April 1996

4. P.B.W. Schwering, Model based feature fusion approach, in 'Detection and Remediation Technologies for Mines and Minelike Targets VI', Proceedings SPIE-Orlando Vol. 4394 paper-125, April 2001 (these proceedings)

5. W. de Jong, H.A. Lensen, Y.H.L. Janssen, Sophisticated test facilin' to delect land mines, in 'Detection and Remediation Technologies for Mines and Minelike Targets IV', Proceedings SPIE-Orlando Vol. 3710 ppl4091418, April 1999

6. L.J. Carter, A. Kokonozi, Landmine detection using a time-sequence of infrared images, JRC, January 2001. internal report.

7. P.A. Jacobs, Thermal Infrared Characterization of Ground Targets and Backgrounds, Tutorial Texts in Optical Engineering Vol. TT 26, 1996 (chapter 3)

8. W. de Jong, A.N. de Jong, Beproevingen van alternatieve mijndetectie methoden, TNO-FEL report FEL-99-A105. February 2000 (text in Dutch)

9. L.J. Carter, G.H.B. Bryant, M. Le Fevre, W.C. Wong, Moisture and Landmine detection, in 'Proceedings EUREL/IEE International Conference on the Detection of Abandoned Landmines (MD96)', Edinburgh, IEE Conference Publication Number 431, pp83-87, 1996

10. S.M. Khanna, F. Paquet, R. Apps, J.S. Seregelyi, New hybrid remote sensing method using HPM illumination/R detection for mine detection, , in 'Detection and Remediation Technologies for Mines and Minelike Targets III'. Proceedings SPIE-Orlando Vol. 3392, April 1998

11. H.A. Lensen, P.B.W. Schwering, G. Rodríguez Marín, J.M.H. Hendrickx, Measurements and modeling of soil water distribution around landmines in natural soil, in 'Detection and Remediation Technologies for Mines and Minelike Targets VI', Proceedings SPIE-Orlando Vol. 4394 paper-50, April 2001 (these proceedings) 